\title{
Gauge invariance of complex general relativity
}

\author{
Merced Montesinos and José David Vergara
}

\begin{abstract}
In this paper it is implemented how to make compatible the boundary conditions and the gauge fixing conditions for complex general relativity written in terms of Ashtekar variables using the approach of Ref. [1]. Moreover, it is found that at first order in the gauge parameters, the Hamiltonian action is (on shell) fully gauge-invariant under the gauge symmetry generated by the first class constraints in the case when spacetime $\mathcal{M}$ has the topology $\mathcal{M}=R \times \Sigma$ and $\Sigma$ has no boundary. Thus, the statement that the constraints linear in the momenta do not contribute to the boundary terms is right, but only in the case when $\Sigma$ has no boundary.
\end{abstract}

Key words: Ashtekar variables. Boundary conditions. Gauge fixing conditions.

It is a common belief that the (internal) gauge symmetry present in Yang-Mills theory is of a different kind with respect to the gauge symmetry involved in generally covariant theories as general relativity. This conclusion comes from the fact that when Yang-Mills theory is expressed in Hamiltonian form, the constraints are linear and homogeneous in their momenta. So, the Hamiltonian action of Yang-Mills theory is fully gauge-invariant under the gauge symmetry generated by the first class constraints. On the other hand, in covariant theories with first class constraints quadratic in their momenta, as general

\footnotetext{
*Department of Physics and Astronomy, University of Pittsburgh, Pittsburgh, PA 15260, USA. Permanent address: Departamento de Física, Centro de Investigación y de Estudios Avanzados del I.P.N., Av. I.P.N. No. 2508, 07000 Ciudad de México, México. e-mail: merced@fis.cinvestav.mx

†Instituto de Ciencias Nucleares, Universidad Nacional Autónoma de México, 70-543, Ciudad de México, México. e-mail: vergara@nuclecu.unam.mx
} 
relativity expressed in terms of Arnowitt-Deser-Misner (ADM) variables, the Hamiltonian action is non gauge-invariant under the gauge symmetry generated by the first class constraints, rather, gauge invariance of the action is broken down [see for instance Ref. [2]]. The cause of this is precisely the quadratic (in the momenta) terms in the constraints. However, in Ref. [1] was shown for generally covariant theories with a finite number of degrees of freedom that both type theories, those with constraints linear and homogeneous in the momenta and those with quadratic and higher order terms in the momenta, are very similar in some sense, the only difference (from the point of view of gauge symmetry) appears from the fact that in covariant theories is necessary to take into account more carefully the boundary terms. To handle the compatibility of the boundary conditions with the gauge fixing conditions, the approach developed in Ref. [1] essentially takes advantage of the gauge symmetry generated by the gauge first class constraints.

In this paper, the programme of Ref. [1] is implemented for self-dual gravity [3] where the first class constraints are quadratic in their momenta. It is very well known that complex general relativity can be rewritten in a Yang-Mills-like form if one uses Ashtekar variables to label the phase space of complex general relativity [3], which opened an avenue for quantizing gravity from a nonperturbative viewpoint [see Refs. [4,5] for a recent overview]. In spite of this success in the quantum theory, some facts still remain unclear even at classical level. For instance, a natural question is whether or not complex general relativity expressed in terms of Ashtekar variables shares the same property as 'usual' Yang-Mills theory, namely, is the Hamiltonian action of complex general relativity in terms of Ashtekar variables fully gauge-invariant? Here, it is shown that the answer is in the affirmative. More precisely, the conclusion is that this Hamiltonian action is (on shell) gauge-invariant. It is important to mention that the techniques of Ref. [1] had not been applied to a generally covariant theory with an infinite number of degrees of freedom, so this contribution fills out this gap in the literature.

In what follows, it is assumed that the spacetime $\mathcal{M}$ has the topology $\mathcal{M}=R \times \Sigma$, and $\Sigma$ has no boundary, and Lorentzian signature is considered too. One starts with the self-dual (anti-self-dual) Hamiltonian form of complex general relativity expressed in terms of Ashtekar variables

$$
S=\int_{x^{0}=x_{i}^{0}}^{x^{0}=x_{f}^{0}} d x^{0} \int_{\Sigma} d^{3} x\left\{\dot{A}_{a}^{i} \widetilde{\Pi}_{i}^{a}-\left(\lambda \widetilde{\widetilde{H}}+\lambda^{i} \widetilde{G}_{i}+\lambda^{a} \widetilde{V}_{a}\right)\right\}
$$

The dependency of the Lagrange multipliers $\underset{\sim}{\lambda}, \lambda^{i}, \lambda^{a}$, and the phase space variables $A_{a}^{i}$, $\widetilde{\Pi}_{i}^{a}$ on the Lagrangian variables is

$$
\begin{aligned}
\lambda & =\frac{\left(N-N^{b} E_{b}^{j} M_{j}\right)}{E\left(1-M^{2}\right)}\left(\frac{4 \pi G}{c^{3} q_{\epsilon}}\right), \\
\lambda^{i} & =-\frac{1}{q_{\epsilon}}\left(\frac{1}{2} \epsilon^{i}{ }_{j k}^{4} \omega_{0}{ }^{j k}-\epsilon i^{4} \omega_{0}{ }^{0} i\right)
\end{aligned}
$$




$$
\begin{aligned}
\lambda^{a} & =N^{a}-\frac{\left(N-N^{b} E_{b}^{j} M_{j}\right)\left(E E_{k}^{a} M^{k}\right)}{E\left(1-M^{2}\right)}, \\
A_{a}^{i} & =\frac{1}{q_{\epsilon}}\left(\frac{1}{2} \epsilon^{i}{ }_{j k}{ }^{4} \omega_{a}{ }^{j k}-\epsilon i^{4} \omega_{a}{ }^{\hat{0} i}\right), \\
\widetilde{\Pi}_{i}^{a} & =\left(\frac{c^{3} i \epsilon q_{\epsilon}}{8 \pi G}\right) E\left(E_{i}^{a}+\epsilon i \epsilon_{i}{ }^{j k} M_{j} E_{k}^{a}\right) .
\end{aligned}
$$

$\epsilon=1(\epsilon=-1)$ corresponds with the self-dual (anti-self-dual) action. In the foliation it has been used the splitting of the (inverse) tetrad field $e^{\hat{0}}=N d x^{0}+M_{i} E_{a}^{i} d x^{a}, e^{i}=E_{a}^{i} N^{a} d x^{0}+$ $E_{a}^{i} d x^{a}$. Also, $E=\operatorname{det} E_{a}^{i}, E_{i}^{a}$ is the inverse of $E_{a}^{i} ; M^{2}=M_{i} M^{i}$ with $M^{i}=M_{j} \delta^{j i}$. The constraints have the form

$$
\begin{aligned}
\widetilde{\widetilde{H}} & =\epsilon^{i j k} \widetilde{\Pi}_{i}^{a} \widetilde{\Pi}_{j}^{b} F_{a b k}(A), \\
\widetilde{G}_{i} & =\mathcal{D}_{a} \widetilde{\Pi}_{i}^{a}=\partial_{a} \widetilde{\Pi}_{i}^{a}-q_{\epsilon} \epsilon_{i j}{ }^{k} A_{a}^{j} \widetilde{\Pi}_{k}^{a}, \\
\widetilde{V}_{a} & =\widetilde{\Pi}_{i}^{b} F_{a b}{ }^{i}(A),
\end{aligned}
$$

with $F_{a b}{ }^{i}(A)=\partial_{a} A_{b}^{i}-\partial_{b} A_{a}^{i}-q_{\epsilon} \epsilon^{i}{ }_{j k} A_{a}^{j} A_{b}^{k}$ the curvature of $A_{a}^{i}$. The symplectic structure of the phase space is given by

$$
\begin{aligned}
& \left\{\widetilde{\Pi}_{i}^{a}(x), \widetilde{\Pi}_{j}^{b}(y)\right\}=0, \\
& \left\{A_{a}^{i}(x), \widetilde{\Pi}_{j}^{b}(y)\right\}=\delta_{a}^{b} \delta_{j}^{i} \delta^{3}(x, y), \\
& \left\{A_{a}^{i}(x), A_{b}^{j}(y)\right\}=0,
\end{aligned}
$$

where $a$ is a spatial index and runs over $1,2,3$; the index $i$ runs over the Lie algebra of $S L(2, C)$. The self-dual (anti-self-dual) coupling constants are chosen as $q_{\epsilon}=-1$ as usual. Sometimes the 'time gauge' is chosen too, $M_{i}=0$, which simplifies the definitions of the phase space variables. This can be done but it is not necessarily in principle. Up to here, it all has concerned with the first order formalism. It is possible to make contact with the second order formalism. Assuming there is no torsion and using $0=\nabla_{\alpha} e_{\beta}^{I}=$ $\partial_{\alpha} e_{\beta}^{I}-{ }^{4} \omega_{\alpha J}{ }^{I} e_{\beta}^{J}+A_{\alpha \beta}{ }^{\gamma} e_{\gamma}^{I}$ follows $\partial_{[a} E_{b]}^{i}-{ }^{4} \omega_{[a \hat{0}}{ }^{i} E_{b]}^{j} M_{j}+\epsilon^{i}{ }_{j k}{ }^{4} \Gamma_{[a}^{j} E_{b]}^{k}$ with ${ }^{4} \Gamma_{a}^{i}=-\frac{1}{2} \epsilon^{i}{ }_{j k}{ }^{4} \omega_{a}{ }^{j k}$ [see the definition of $A_{a}^{i}$. When the 'time gauge' is fixed, $M_{i}=0$, and then ${ }^{4} \Gamma_{a}^{i}=\Gamma_{a}^{i}$ with $\Gamma_{a}^{i}$ given by $\partial_{[a} E_{b]}^{i}+\epsilon^{i}{ }_{j k} \Gamma_{[a}^{j} E_{b]}^{k}=0$.

Due to the fact that the algebra of constraint closes with the diffeomorphism constraint $\widetilde{D}_{a}=\left(\widetilde{\Pi}_{i}^{b} F_{a b}{ }^{i}-A_{a}^{i} \widetilde{G}_{i}\right)$ instead of the vector constraint $\widetilde{V}_{a}$, and because one is interested in computing the effect of the gauge symmetry generated by the first class constraints on the action, it will be considered from now on the Hamiltonian action

$$
S=\int_{x^{0}=x_{i}^{0}}^{x^{0}=x_{f}^{0}} d x^{0} \int_{\Sigma} d^{3} x\left\{\dot{A}_{a}^{i} \widetilde{\Pi}_{i}^{a}-\left(\underset{\sim}{\widetilde{H}}+\lambda^{i} \widetilde{G}_{i}+\lambda^{a} \widetilde{D}_{a}\right)\right\}
$$


instead of Eq. (1).

The diffeomorphism constraint $\widetilde{D}_{a}$ as well as the Gauss constraint are linear in the momentum $\widetilde{\Pi}_{i}^{a}$. Therefore, one can try to implement for gravity the ideas of Ref. [1]. Due to the fact that the Hamiltonian constraint $\widetilde{\widetilde{H}}$ is quadratic in the momenta $\widetilde{\Pi}_{i}^{a}$, it could be expected that a (temporal) boundary term should be added to the action of Eq. (6) to get back a fully covariant action for complex general relativity. Following Refs. [2, [1], the change on the action of Eq. (6) generated by the Hamiltonian, diffeomorphism and Gauss constraints is to order $\varepsilon$

$$
S^{\prime}=S\left[A^{\prime}, \widetilde{\Pi}^{\prime}, \lambda^{\prime}, \lambda^{\prime i}, \lambda^{\prime a}\right]=S\left[A, \widetilde{\Pi}, \underset{\sim}{\lambda} \lambda^{i}, \lambda^{a}\right]+\left.\delta_{\varepsilon} B\right|_{x^{0}=x_{i}^{0}} ^{x^{0}=x_{0}^{0}},
$$

with

$$
\begin{aligned}
& A^{\prime i}=A_{a}^{i}+2 \underset{\sim}{\varepsilon} \epsilon^{i j k} \widetilde{\Pi}_{j}^{b} F_{a b k}-\mathcal{D}_{a} \varepsilon^{i}+\mathcal{L}_{\vec{\varepsilon}} A_{a}^{i}, \\
& \widetilde{\Pi}_{i}^{a}=\widetilde{\Pi}_{i}^{a}+2\left[\partial_{b}\left(\underset{\sim}{\varepsilon} \epsilon_{i}^{j k} \widetilde{\Pi}_{j}^{b} \widetilde{\Pi}_{k}^{a}\right)+\epsilon_{i m}^{n} A_{b}^{m}\left(\varepsilon_{\sim} \epsilon_{n}{ }^{j k} \widetilde{\Pi}_{j}^{b} \widetilde{\Pi}_{k}^{a}\right)\right] \\
& +\epsilon_{i j}{ }^{k} \varepsilon^{j} \widetilde{\Pi}_{k}^{a}+\mathcal{L}_{\vec{\varepsilon}} \widetilde{\Pi}_{i}^{a}, \\
& \lambda^{\prime}=\underset{\sim}{\lambda}+\underset{\sim}{\dot{\sim}}+\mathcal{L}_{\vec{\varepsilon}} \underset{\sim}{\lambda}-\mathcal{L}_{\vec{\lambda}} \varepsilon \\
& \lambda^{\prime i}=\lambda^{i}+\dot{\varepsilon}^{i}+4 \widetilde{\Pi}_{j}^{a} \widetilde{\Pi}^{b j}\left(\underset{\sim}{\lambda} \partial_{b} \varepsilon-\underset{\sim}{\varepsilon} \partial_{b} \lambda\right) A_{a}^{i}-[\lambda, \varepsilon]^{i}+\mathcal{L}_{\vec{\varepsilon}} \lambda^{i}-\mathcal{L}_{\vec{\lambda}} \varepsilon^{i}, \\
& \lambda^{\prime a}=\lambda^{a}+\dot{\varepsilon}^{a}+4 \widetilde{\Pi}_{j}^{a} \widetilde{\Pi}^{b j}\left(\underset{\sim}{\lambda} \partial_{b} \varepsilon-\varepsilon \partial_{b} \lambda\right)-[\lambda, \varepsilon]^{a}, \\
& \delta_{\varepsilon} B=\int d^{3} x \widetilde{\Pi}_{i}^{a}(x) \frac{\delta}{\delta \widetilde{\Pi}_{i}^{a}(x)} \int d^{3} y\left\{\underset{\sim}{\widetilde{H}}+\varepsilon^{i} \widetilde{G}_{i}+\varepsilon^{a} \widetilde{D}_{a}\right\} \\
& -\int d^{3} x\left\{\varepsilon \widetilde{\widetilde{H}}+\varepsilon^{i} \widetilde{G}_{i}+\varepsilon^{a} \widetilde{D}_{a}\right\}
\end{aligned}
$$

Here, $\mathcal{L}_{\vec{\varepsilon}} A_{a}^{i}=\varepsilon^{b} \partial_{b} A_{a}^{i}+A_{b}^{i} \partial_{a} \varepsilon^{b}, \mathcal{L}_{\vec{\varepsilon}} \widetilde{\Pi}_{i}^{a}=\varepsilon^{b} \partial_{b} \widetilde{\Pi}_{i}^{a}-\widetilde{\Pi}_{i}^{b} \partial_{b} \varepsilon^{a}+\widetilde{\Pi}_{i}^{a} \partial_{b} \varepsilon^{b},[\lambda, \varepsilon]^{i}=\epsilon^{i}{ }_{j k} \lambda^{j} \varepsilon^{k}$, $[\lambda, \varepsilon]^{a}=\mathcal{L}_{\vec{\lambda}} \varepsilon^{a}$. By inserting in $\delta_{\varepsilon} B$ the expressions of the Hamiltonian, diffeomorphism, and Gauss constraints, it can be seen immediately that the contribution of both diffeomorphism and Gauss constraints is vanishing. Therefore, the boundary term $B$ is determined by the Hamiltonian constraint only, and is given by

$$
\delta_{\varepsilon} B=\int d^{3} x \widetilde{\Pi}_{i}^{a}(x) \frac{\delta}{\delta \widetilde{\Pi}_{i}^{a}(x)} \int d^{3} y \underset{\sim}{\varepsilon \widetilde{H}}-\int d^{3} x \underset{\sim}{\varepsilon} \widetilde{\widetilde{H}}=\int d^{3} x \underset{\sim}{\varepsilon \widetilde{\widetilde{H}}} .
$$

Thus,

$$
S^{\prime}=S\left[A^{\prime}, \widetilde{\Pi}^{\prime}, \lambda^{\prime}, \lambda^{\prime i}, \lambda^{\prime a}\right]=S\left[A, \widetilde{\Pi}, \lambda, \lambda^{i}, \lambda^{a}\right]+\left.\int d^{3} x \underset{\sim}{\varepsilon \widetilde{\widetilde{H}}}\right|_{x^{0}=x_{i}^{0}} ^{x^{0}=x_{0}^{0}}
$$

What is surprisingly is that $S^{\prime}$ and $S$ differ by a (temporal) surface term involving the Hamiltonian constraint only. Now, the Dirac formalism [6 establishes first class constraints weakly vanish at any time $x^{0}$, which means the boundary term in Eq. (10) 
vanishes 'on shell'. Therefore, the action of Eq. (6) is (on shell) fully gauge-invariant under the gauge symmetry generated by the first class constraints. Some comments are in order. First, general relativity in terms of Ashtekar variables shares the same property as standard Yang-Mills theory, in the sense that gauge invariance is not drop at the (temporal) boundary, even though complex general relativity has a constraint quadratic in their momenta. In Yang-Mills theory, on the other hand, the action is fully gauge-invariant and not only 'on shell' due to the fact that the constraints are linear in the momenta. Whether or not this similarity between general relativity and Yang-Mills theory is a deep one deserves to be investigated. For the present purposes, it has been exhibited only that complex general relativity is truly 'close' to a Yang-Mills theory in the sense explained. This property of the Hamiltonian action of complex general relativity in terms of Ashtekar variables is not present even in simple models with finite degrees of freedom, where $S^{\prime}$ and $S$ differ by a (temporal) term which is not proportional to the quadratic constraints $\mathrm{f}$.

Now, it is time to go to the issue concerning the compatibility of the boundary conditions and the gauge fixing conditions. For the action $S\left[A, \widetilde{\Pi}, \lambda, \lambda^{i}, \lambda^{a}\right]$ the boundary conditions are

$$
\begin{aligned}
& A_{a}^{i}\left(x_{i}^{0}, x^{a}\right)=\mathcal{A}_{a}^{i}\left(x^{a}\right), \\
& A_{a}^{i}\left(x_{f}^{0}, x^{a}\right)=\mathcal{B}_{a}^{i}\left(x^{a}\right),
\end{aligned}
$$

where $\mathcal{A}_{a}^{i}\left(x^{a}\right)$, and $\mathcal{B}_{a}^{i}\left(x^{a}\right)$ are smooth configurations of the connection field at the end points. These boundary conditions have to be compatible with the gauge fixing conditions in order to have a well-defined dynamics. The gauge fixing conditions, in general, are expressions of the form

$$
\begin{aligned}
\chi\left(A_{a}^{i}, \widetilde{\Pi}_{i}^{a}, x^{0}, x^{a}\right)=0, & \\
\chi_{a}\left(A_{a}^{i}, \widetilde{\Pi}_{i}^{a}, x^{0}, x^{a}\right) & =0, \quad a=1,2,3, \\
\chi_{i}\left(A_{a}^{i}, \widetilde{\Pi}_{i}^{a}, x^{0}, x^{a}\right) & =0, \quad i=1,2,3 .
\end{aligned}
$$

Suppose, for the moment, they were non compatible with the boundary conditions (11). Under this assumption, dynamics of the gravitational field would be in trouble. How to

\footnotetext{
${ }^{\ddagger}$ For instance, in the case of the parameterized harmonic oscillator, the action $S=\int d \tau\{\dot{x} p+$ $\left.\dot{t} p_{t}-\lambda\left(p_{t}+\frac{p^{2}}{2 m}+\frac{1}{2} m \omega^{2} x^{2}\right)\right\}$ is not fully gauge-invariant under the finite gauge transformations of the phase space variables and the Lagrange multiplier generated by the first class constraint $H=$ $p_{t}+\frac{p^{2}}{2 m}+\frac{1}{2} m \omega^{2} x^{2}$, rather, under the gauge symmetry the action transforms as $S\left[x^{\prime}, t^{\prime}, p^{\prime}, p^{\prime}, \lambda^{\prime}\right]=$

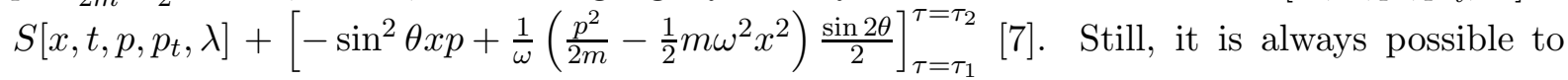
build a fully covariant action by adding a suitable (temporal) boundary term. This new action is $S_{i n v}=S-\left.\frac{1}{2} x p\right|_{\tau_{i}} ^{\tau_{f}}$. Notice that the added boundary term is not proportional to the first class constraint $H$. In the ADM formalism of general relativity a similar result appears [2].
} 
make compatible both things? One possibility is to use the approach of Ref. [1]. The idea developed in Ref. [1] is to take advantage of the gauge symmetry generated by the first class constraints. Instead of considering the action $S\left[A, \widetilde{\Pi}, \lambda \underset{\sim}{\sim} \lambda^{i}, \lambda^{a}\right]$, it has to be taken into account a new action $S_{i n v}$, related to $S\left[A, \widetilde{\Pi}, \lambda, \lambda^{i}, \lambda^{a}\right]$, through the gauge symmetry [1]. This action is

$$
S_{\text {first order }}\left[A, \widetilde{\Pi}, \underset{\sim}{\sim} \lambda^{i}, \lambda^{a}, \varepsilon\right]=S\left[A, \widetilde{\Pi}, \underset{\sim}{\sim} \lambda^{i}, \lambda^{a}\right]+\left.\int d^{3} x \underset{\sim}{\varepsilon \widetilde{\widetilde{H}}}\right|_{x^{0}=x_{i}^{0}} ^{x^{0}=x_{0}^{0}}
$$

Taking into account Eq. (8), the RHS of Eq. (13) can be written as

$$
\begin{aligned}
& S_{\text {first order }}\left[A, \widetilde{\Pi}, \underset{\sim}{\sim} \lambda^{i}, \lambda^{a}, \varepsilon\right]=S\left[A, \widetilde{\Pi}, \lambda, \lambda^{i}, \lambda^{a}\right]+\left.\int d^{3} x \underset{\sim}{\underset{\sim}{\tilde{H}}}\right|_{x^{0}=x_{i}^{0}} ^{x^{0}=x_{0}^{0}}, \\
& =\int_{x^{0}=x_{i}^{0}}^{x^{0}=x_{f}^{0}} d x^{0} \int_{\Sigma} d^{3} x\left\{\dot{A}^{\prime}{ }_{a}^{i}{\widetilde{\Pi^{\prime}}}_{i}^{a}-\left(\lambda^{\prime} \widetilde{\widetilde{H}}^{\prime}+\lambda^{\prime i}{\widetilde{G^{\prime}}}_{i}+\lambda^{\prime a}{\widetilde{D^{\prime}}}_{a}{ }_{a}\right)\right\}, \\
& =S\left[A^{\prime}, \widetilde{\Pi^{\prime}}, \lambda^{\prime}, \lambda^{\prime i}, \lambda^{\prime a}\right] \text {. }
\end{aligned}
$$

From the last line of Eq. (14) the action $S_{\text {first order }}\left[A, \widetilde{\Pi}, \underset{\sim}{\sim} \lambda^{i}, \lambda^{a}, \varepsilon\right]$ can be considered a functional of the gauge related phase space variables $A^{\prime i}$ and $\widetilde{\Pi}_{i}^{\prime}{ }_{i}$ and of the Lagrange multipliers ${\underset{\sim}{\prime}}^{\prime}, \lambda^{\prime}$, and $\lambda^{\prime a}$. Keeping this in mind it is pretty obvious that the boundary conditions for $S_{\text {first order }}\left[A, \widetilde{\Pi}, \lambda, \lambda^{i}, \lambda^{a}, \varepsilon\right]$ are

$$
\begin{aligned}
& {A^{\prime}}_{a}^{i}\left(x_{i}^{0}, x^{a}\right)=\mathcal{A}_{a}^{\prime i}\left(x^{a}\right), \\
& A^{\prime i}\left(x_{f}^{0}, x^{a}\right)=\mathcal{B}_{a}^{\prime i}\left(x^{a}\right),
\end{aligned}
$$

where $\mathcal{A}_{a}^{\prime i}\left(x^{a}\right)$, and $\mathcal{B}_{a}^{\prime}{ }_{a}\left(x^{a}\right)$ are the initial (at $x_{i}^{0}$ ) and final (at $x_{f}^{0}$ ) configurations of the gauge related connection field $A_{a}^{\prime}$. Instead of considering the variational principle defined by the action $S_{\text {first order }}\left[A, \widetilde{\Pi}, \lambda, \lambda^{i}, \lambda^{a}, \varepsilon\right]$ in the form given by the last line in the RHS of Eq. (14) together with the boundary conditions of Eq. (15), it is possible to go back to the original set of variables. There, the phase space variables are $A_{a}^{i}$ and $\widetilde{\Pi}_{i}^{a}$, the action is given by Eq. (13) and its boundary conditions, using Eq. (8), are

$$
\begin{aligned}
& \left(A_{a}^{i}+2 \varepsilon_{\sim} \epsilon^{i j k} \widetilde{\Pi}_{j}^{b} F_{a b k}-\mathcal{D}_{a} \varepsilon^{i}+\mathcal{L}_{\vec{\varepsilon}} A_{a}^{i}\right)\left(x_{i}^{0}, x^{a}\right)=\mathcal{A}_{a}^{i}\left(x^{a}\right), \\
& \left(A_{a}^{i}+2 \varepsilon \epsilon^{i j k} \widetilde{\Pi}_{j}^{b} F_{a b k}-\mathcal{D}_{a} \varepsilon^{i}+\mathcal{L}_{\vec{\varepsilon}} A_{a}^{i}\right)\left(x_{f}^{0}, x^{a}\right)=\mathcal{B}_{a}^{\prime i}\left(x^{a}\right) .
\end{aligned}
$$

This new variational principle, composed by the new action (13) and the new boundary conditions (16), has two peculiarities. First, the action $S_{\text {first order }}\left[A, \widetilde{\Pi}, \lambda, \lambda^{i}, \lambda^{a}, \varepsilon\right]$ is 'invariant' in the sense explained, namely, in terms of the gauge related variables the action $S_{\text {first order }}$ is the original action $S$ but written in terms of the gauge related variables [see the RHS of Eq. (14)]. Second, the new action (13) also allows to make compatible 
the boundary conditions (16) with the gauge fixing conditions (12), through the gauge parameters involved there [i]]. To do this, it has to be plugged into the gauge conditions (12) the expressions of the phase space variables $A_{a}^{i}$ and $\widetilde{\Pi}_{i}^{a}$ in terms of the gauge related variables ${A^{\prime}}_{a}^{i}, \widetilde{\Pi}_{i}^{a}$ as well as of the gauge parameters $\underset{\sim}{\varepsilon}, \varepsilon^{i}$, and $\varepsilon^{a}$. From these equations, one needs to solve for the gauge parameters ${\underset{\sim}{\varepsilon},}^{i}$, and $\varepsilon^{a}$ in terms of the gauge related phase space variables $A_{a}^{\prime i}$, and ${\widetilde{\Pi^{\prime}}}_{i}^{a}$ and plug them into the RHS of Eq. (13) and Eq. (16). Of course, 'on shell' there is no reason to plug these expressions for the gauge parameters into the RHS of Eq. (13) because $S_{\text {first order }}=S$ 'on shell'. Notice that in the boundary conditions it will appear $A_{a}^{\prime i}\left(x_{i}^{0}, x^{a}\right)\left[=\mathcal{A}_{a}^{i}\left(x^{a}\right)\right], A_{a}^{\prime i}\left(x_{f}^{0}, x^{a}\right)\left[=\mathcal{B}_{a}^{\prime i}\left(x^{a}\right)\right], \widetilde{\Pi}_{i}^{a}\left(x_{i}^{0}, x^{a}\right)$, and ${\widetilde{\Pi^{\prime}}}_{i}^{a}\left(x_{f}^{0}, x^{a}\right)$. They play the role of 'parameters'. In addition, $\mathcal{A}_{a}^{\prime i}\left(x^{a}\right) \neq \mathcal{A}_{a}^{i}\left(x^{a}\right)$ and $\mathcal{B}^{\prime i}{ }_{a}^{i}\left(x^{a}\right) \neq \mathcal{B}_{a}^{i}\left(x^{a}\right)$ in general. There is no reason why they should be the same expressions. As opposed to here, in Ref. [1], the equality among them was adopted.

In summary, the method of Ref. [1] is totally systematic and can be applied to complex general relativity in terms of Ashtekar variables without any problems. The well-defined variational principle features i) an action defined by the RHS of (13), ii) boundary conditions given by (16) where the gauge parameters are in terms of the gauge related variables ('parameters') iii) these boundary conditions are compatible with the gauge fixing conditions (12). So, the method of Ref. [1] can also be applied to any generally covariant field theory with the obvious modifications required in theories with an infinite number of degrees of freedom, as in the case of general relativity studied here. Of course, the present analysis has been restricted to space times $\mathcal{M}$ having the topology $\mathcal{M}=R \times \Sigma$, where $\Sigma$ has no boundary. Before concluding, three more comments concerning Barbero variables, Husain-Kuchar's model, and spatial boundaries for $\Sigma$. First, what would have happened if one had used Barbero variables instead of Ashtekar variables? In terms of Barbero variables the Hamiltonian constraint is modified by a non polynomial contribution in the phase space variables [ [8]

$$
\widetilde{\widetilde{H}}=\epsilon^{i j k} \widetilde{E}_{i}^{a} \widetilde{E}_{j}^{b} F_{a b k}(A)+\frac{2\left(\beta^{2}+1\right)}{\beta^{2}} \widetilde{E}_{[i}^{a} \widetilde{E}_{j]}^{b}\left(A_{a}^{i}-\Gamma_{a}^{i}\right)\left(A_{b}^{j}-\Gamma_{b}^{j}\right) .
$$

Obviously, in Barbero case, the difference between $S^{\prime}$ and $S$ is not proportional to the Hamiltonian constraint, and the action $S$ is not 'on shell' gauge-invariant in this case. Nevertheless, following Ref. [1], is possible to add a suitable boundary term to $S$ to build a fully gauge-invariant action. No specific proposal for this boundary term is presented in this paper, but it is possible to build it in principle. Second, it is important to notice that the Husain-Kuchar model, which is 'close' to complex general relativity in terms of Ashtekar variables, is fully gauge-invariant under the gauge symmetry generated by the diffeomorphism and Gauss constraints in the case when $\mathcal{M}$ has the topology $\mathcal{M}=R \times \Sigma$ and $\Sigma$ has no boundary. This is so because in that model the Hamiltonian constraint is missing [9]. Finally, the issue of the gauge invariance of complex general relativity when $\Sigma$ has boundaries deserves to be studied. Due to the fact that diffeomorphism constraint 
'moves' spatial boundaries, it is natural to expect to add a boundary term to get back a fully covariant action, namely

$$
S_{i n v}=S-\left.\int_{\Sigma} d^{3} x C\left(\widetilde{\Pi}_{i}^{a}, A_{a}^{i}\right)\right|_{x^{0}=x_{i}^{0}} ^{x^{0}=x_{0}^{0}}-\int_{x^{0}=x_{i}^{0}}^{x^{0}=x_{f}^{0}} d x^{0} \int_{\partial \Sigma} I\left(\widetilde{\Pi}_{i}^{a}, A_{a}^{i}\right) d S .
$$

The study of this issue is left for future work.

\section{ACKNOWLEDGMENTS}

Warm thanks to Jerzy Plebański and Joseph Samuel for their valuable comments. MM thanks financial support provided by the Sistema Nacional de Investigadores (SNI) of the Secretaría de Educación Pública (SEP) of Mexico. The summer stay of MM at the Department of Physics and Astronomy of the University of Pittsburgh, where this paper was finished, is supported by the Mexican Academy of Sciences and The United StatesMexico Foundation for Science. Also MM thanks all the members of the Department of Physics and Astronomy of the University of Pittsburgh for their warm hospitality. JDV is partially supported by grants DGAPA-UNAM IN100397 and CONACyT 32431-E. 


\section{REFERENCES}

[1] Henneaux M and Vergara J D, in Sakharov Memorial Lectures in Physics, edited by Keldysh L V and Fainberg V Ya (Nova Science Publishers Inc.: New York, 1992) p. 111; Henneaux M, Teitelboim C and Vergara J D, Nucl. Phys. B 387, 391 (1992).

[2] Teitelboim C, Phys. Rev. D 25, 3159 (1982).

[3] Plebański J F, J. Math. Phys. 18, 2511 (1977); Ashtekar A, Phys. Rev. Lett. 57, 2244 (1986), Phys. Rev. D 36, 1587 (1987); Jacobson T and Smolin L, Phys. Lett. B 196, 39 (1987), Class. Quantum Grav. 5, 583 (1988); Samuel J, Pramana J. Phys. 28, L429 (1989); Capovilla R, Dell J, Jacobson T and Mason L, Class. Quantum Grav. 8, 41 (1991).

[4] Rovelli C, 'Strings, loops and others: a critical survey of the present approaches to quantum gravity', in Gravitation and Relativity at the Turn of the Millennium, edited by Dadhich N and Narlikar J (Inter University Centre for Astronomy and Astrophysics: India, 1998).

[5] Rovelli C, J. Math. Phys. 41, 3776 (2000).

[6] Dirac P A M, Lectures on Quantum Mechanics (Belfer Graduate School of Science, New York, 1964).

[7] Montesinos M, 'Relational evolution of the degrees of freedom of generally covariant quantum theories' Gen. Rel. Grav. (accepted for publication), gr-qc/0002023.

[8] Barbero J F, Phys. Rev. D 51, 5507 (1995).

[9] Husain V and Kuchar K, Phys. Rev. D 42, 4070 (1990). 